\title{
Psychometric Evaluation of a Barriers to Mental Health Treatment Questionnaire for Latina/o/x Caregivers of Children and Adolescents
}

\author{
Alejandro L. Vázquez ${ }^{1}$ (D) Demi Culianos ${ }^{1}$ (D) Cynthia M. Navarro Flores ${ }^{1}$ (D) \\ María de la Caridad Alvarez ${ }^{1}$ (D) . Tyson S. Barrett ${ }^{1}$ D. \\ Melanie M. Domenech Rodríguez ${ }^{1}$
}

Accepted: 24 September 2021 / Published online: 7 October 2022

(c) The Author(s), under exclusive licence to Springer Science+Business Media, LLC, part of Springer Nature 2021

\begin{abstract}
Background Knowledge regarding barriers faced by Latina/o/x caregivers in accessing youth mental health services (MHS) have largely depended on resource intensive interviewbased assessments.

Objective We evaluated a questionnaire for Latina/o/x caregivers of youths that presents a briefer and more feasible alternative.

Method We conducted a psychometric evaluation of the Barriers to Treatment Questionnaire - Latina/o/x Caregivers (BTQ-LC) with a sample of 598 Latina/o/x caregivers from across the United States. Descriptive statistics and confirmatory factor analyses were used to identify common barriers to services, confirm the factor structure of the scale, and establish construct validity.

Results Descriptive statistics suggest that not knowing where and how to access services, and normalization of youth psychopathology were the most frequently reported barriers among caregivers of youth with clinically elevated problems on the CBCL. Confirmatory factor analysis suggests that the BTQ-LC was best represented by a three-factor structure: (1) structural, (2) perceptions regarding mental health problems, and (3) services. Our finding suggest that the BTQ-LC could also be used as a single factor as fit indices ranged from acceptable to poor. BTQ-LC scales were all negatively correlated with the utilization of common youth MHS (i.e., psychological counseling, medical doctors, school professionals). Conclusions The BTQ-LC represents an important step towards improving our understanding and assessment of barriers to services contributing to mental health disparities among Latina/o/x youths.
\end{abstract}

Keywords Latina/o/x $\cdot$ Mental Health Services $\cdot$ Barriers $\cdot$ Caregivers $\cdot$ Youths

Alejandro L. Vázquez

Alejandro.Vazquez@aggiemail.usu.edu

1 Department of Psychology, Utah State University, 2810 Old Main Hill, 84322 Logan, UT, USA 


\section{Introduction}

Latina/o/x youths experience significant mental health disparities within the United States (Merikangas et al. 2011). Research suggests that families of color are disproportionately impacted by barriers to accessing youth mental health services (MHS), which contributes to underutilization of services (Malhotra et al. 2015). While caregivers are important gatekeepers to accessing youth services, few studies have specifically examined within group factors impeding Latina/o/x caregivers from accessing youth MHS (Reardon et al. 2017; Villagrana 2010). Much of the service utilization literature has focused on White and Black caregivers' barriers in accessing youth MHS or examinations of between group differences (Reardon et al. 2017; Tsang et al. 2020). This may be in part due to a lack of validated research tools designed to efficiently gather information regarding barriers faced by Latina/o/x caregivers. Research often depends on measures that exclude important influences on help-seeking within communities of color, have not been validated, or require resource intensive methods that may reduce the viability of collecting large sample sizes required for quantitative analyses (Ascher et al. 1996; Berger-Jenkins et al. 2012; Chapman and Stein 2014; Gerdes et al. 2014). We address this need by validating a brief barriers-to-treatment questionnaire to study factors impeding Latina/o/x caregivers from accessing youth MHS.

Theoretical frameworks of MHS utilization suggest that families' decisions to engage their youth in therapy begin with problem recognition (i.e., clinical need, caregiver perceived need), which informs subsequent decisions to seek and engage in formal MHS (Cauce et al. 2002). Families weigh a variety of factors (e.g., finances, transportation, social support, caregiver functioning, insurance) when determining whether formal intervention is feasible and needed (Srebnik et al. 1996). These influences have a cumulative effect with a greater number of barriers being associated with lower odds of utilizing youth MHS (Reardon et al. 2017).

Latina/o/x caregivers encounter a wide array of barriers that impact their willingness and ability to access youth MHS. Common barriers to youth MHS among Latina/o/x families include transportation, income, health insurance coverage, language barriers, difficulty scheduling appointments, fear of discrimination (i.e., race/ethnicity), deportation, and shame or embarrassment associated with mental health stigma (Bridges et al. 2012; Kapke and Gerdes 2016; Kataoka et al. 2002; Scheppers et al. 2006). Latina/o/x caregivers often report fears of being judged or blamed by mental health professional for their child's problems (De Silva et al. 2020) and may also be more likely to normalize their youth's mental health problems relative to White caregivers (Raglin Bignall et al. 2015). Qualitative research on factors impacting youth MHS utilization among Latina/o/x caregivers suggest that families often report mistrust of mental health providers, disagreement with recommendations or diagnoses, fear social rejection associated with their child's problems, lack time for services, and express difficulties with accepting help from mental health professionals (Gerdes et al, 2014). Other qualitative research by Chapman and Stein (2014) has found that even when Latina/o/x caregivers perceive a need for professional intervention, they often report not knowing how to find and access youth MHS in the United States. While barriers to youth MHS among Latina/o/xs are well documented, researchers commonly focus on specific clusters of barriers (e.g., practical, cultural, or perceptions) precluding a broader comparison between factors impacting help-seeking behaviors. 
Barriers may be categorized into broader categories that assist in quantifying the impact of these influences on youth MHS utilization. Owens and colleagues (2002) organized caregivers' youth MHS barriers into three sub-types. Structural barriers are characteristics of the MHS agencies or providers (e.g., availability of providers, transportation, insurance coverage, affordability, accommodation for language and preference of therapist). Perceptions regarding mental health problems include whether caregivers identify their child's problems as warranting formal MHS, perceptions regarding the severity of the youths' problems, and desire to address things on their own. Perceptions of MHS include fears of stigma, shame, discrimination, and lack of satisfaction with prior youth MHS. These barriers directly impact caregivers' treatment engagement and utilization patterns (Srebnik et al. 1996).

Studying specific ethnic groups can uncover knowledge that contributes to universal truths (Cauce 2011). Indeed, research has examined within group factors impacting youth MHS utilization among Latina/o/x families using interview-based measures or open-ended questions with a small number of participants, which has provided a nuanced understanding of barriers to care (Chapman and Stein 2014; De Silva et al. 2020; Gerdes et al. 2014). Here we try to improve the generalizability of research findings by offering a self-report measure. Established questionnaires exist, yet they lack questions assessing important factors among Latina/o/x families that impact MHS utilization (i.e., stigma, shame, and fears of discrimination and deportation; Seid et al. 2009; Kazdin et al. 1997), focus on barriers to treatment retention rather than accessing services (i.e., Kazdin et al. 1997), or were primarily designed for psychiatric services and include factors that may be less relevant to children (e.g., agism scale; Pepin et al. 2009). Furthermore, established barriers questionnaires range from 39 to 56 items presenting a potential burden to research participants that could be alleviated with a briefer measure (Kazdin et al. 1997; Pepin et al. 2009; Seid et al. 2009).

There are several limitations to existing research on barriers to youth MHS. For example, established measures of MHS barriers often focus on clinical samples, yet a systematic review highlighted a need to validate measures with non-clinical samples (Reardon et al. 2017). Additionally, examinations of caregiver reported barriers to youth MHS utilization has predominately focused on between group differences or on specific racial/ethnic groups (i.e., Black and White caregivers; Reardon et al. 2017). We expand existing scholarship by adding information on non-clinical samples and Latina/o/x caregivers specifically.

We adapted the Barriers to Treatment Questionnaire (BTQ; Marques et al. 2010) to assess impediments to accessing youth MHS as reported by Latina/o/x caregivers. The BTQ questionnaire was based on barriers derived from the service utilization literature and has been used to assess challenges in accessing MHS among individuals with obsessive-compulsive symptoms/disorder (Marques et al. 2010; Williams et al. 2012). We choose the BTQ for this adaptation because it included fewer items than established measures, focused on accessing services, and included a wide variety of barriers known to impede Latina/o/x caregivers utilization of youth MHS (i.e., logistic/financial; stigma, shame, and discrimination; treatment perception and satisfaction barriers; Kazdin et al. 1997; Pepin et al. 2009; Reardon et al. 2017; Seid et al. 2009). The measure has also been shown to have good internal consistency among White $(\alpha=0.71)$ and Black $(\alpha=0.82)$ individuals providing optimism that it might perform well within Latina/o/x samples (Marques et al. 2010; Williams et al. 2012). Previous research has used the BTQ as a cumulative score or to provide descriptives regarding common barriers within a populations of interest (Marques et al. 2010; Vázquez et al. 
2021a; Vázquez et al. 2021b; Williams et al. 2012). Although the BTQ's factor structure has not been formally examined, research suggests that the BTQ's content could be organized into a global measure of barriers to MHS and/or subscales (i.e., structural, perceptions of mental health problems, perceptions of MHS; Owens et al. 2002). We sought to establish the factor structure of the adapted BTQ within a sample of Latina/o/x caregivers.

The service utilization literature suggests that a questionnaire is needed to assess Latina/ $\mathrm{o} / \mathrm{x}$ caregivers' barriers to accessing youth MHS. To address this need, we adapted and validated a brief measure assessing caregiver reported barriers to utilizing MHS for Latina/o/x youths. We also described common barriers to seeking youth MHS services among Latina/ $\mathrm{o} / \mathrm{x}$ caregivers. Guided by prior research, we hypothesized that the BTQ would demonstrate a three-factor structure (e.g., structural, perceptions of mental health problems, perceptions of MHS; Owens et al. 2002). We expected that the BTQ-LC global scale and subscales would be negatively correlated with common MHS utilization formats (i.e., psychological counseling, school professionals, medical doctor; Duong et al. 2020). Based on prior research, we expected that caregivers would most frequently report structural barriers as their primary obstacles to youth MHS utilization (i.e., where and how to access services, cost, insurance, transportation; Chapman and Stein 2014; Kapke and Gerdes 2016).

\section{Method}

\section{Procedure and Sample Characteristics}

The current study utilized a national sample of Latina/o/x caregivers of youths 6 to 18 years old recruited through Qualtrics, a survey panel company. Survey panels include individuals that have registered to be contacted regarding research opportunities on an ongoing basis. This sampling method allows researchers to quickly gather data on a national scale from a target population at a reasonable cost (i.e., \$12.50 per participant; Vázquez et al., 2021a). Research has found that survey panels yield high quality data comparable to traditional survey administration practices when validation methods are used (i.e., in-person paper and pencil; Lowry et al. 2016). Abbey and Meloy (2017) describe validation methods as contrasting questions and checks that identify inattentive patterns of responses. Participants that provide incorrect responses to a validation question are likely to be providing poor quality data. Consistent with common practices in survey panel research, Qualtrics provided compensation to participants contingent on completing the entire survey and demonstrating patterns associations with high quality data as identified by validity checks (Kennedy et al. 2020; Lowry et al. 2016). Compensation was in the form of points that participants could redeem for rewards from Qualtrics.

Participants were surveyed between May 21, 2020 and June 18, 2020. Participants were provided a link via email that informed them regarding the nature of the survey, required time, and compensation. Those interested completed a screener. Individuals that met inclusion criteria read information regarding the study and provided informed consent prior to starting the survey. Participants completed the adapted BTQ as part of a 20-min survey collecting information regarding factors impacting youth MHS utilization. If caregivers had multiple children, they were asked to report on the child that presented the most challenges to them as a parent. Responses were required for all survey items. Consistent with best 
practices, we utilized several contrasting validation methods to identify and remove participants that provided low-quality data (i.e., logical statements, directed queries, open-ended queries, response time and pattern, honesty check, response consistency; Abbey and Meloy 2017). Qualtrics also checked internet protocol (IP) addresses to confirm that participants were completing the survey from a computer within the United States and to ensure that respondents only completed the survey once. Approval to conduct the present student was obtained from the Utah State University Institutional Review Board.

To qualify for the current study, participants verified that they were (a) Latina/o/x, (b) a caregiver to at least one youth between the ages 6-18, (c) able to complete the survey in English, and (d) were living in the United States. Among participants that were screened $(n=3,149)$, a third met inclusion criteria $(n=1,128)$. A small number of participants who met inclusion criteria did not provide consent to participate $(n=17)$ and were not administered the survey. The current study did not include data from respondents who provided poor quality information as identified by validity checks $(n=235)$ or those who did not complete the entire survey $(n=278)$. These cases were excluded as Qualtrics only provided data on participants who were compensated for their complete and high-quality work.

The final sample included 598 caregivers of youth between ages 6-18. Caregivers were 38.5 years old on average $(S D=9.1)$, predominantly female $(n=420 ; 70.2 \%)$, were the biological parent $(n=565,94.5 \%)$, varied in educational attainment (less than high school $n=13,0.02 \%$; high school diploma or equivalent $n=185,30.9 \%$; vocational certificate $n=48,8 \%$; associate's $n=103,17.2 \%$; bachelor's $n=180,30.1 \%$, master's $n=65,10.9 \%$; doctorate $n=4,0.7 \%$ ), were citizens of the United States $(n=537,89.8 \%)$, and most frequently reported a household income between $\$ 30,000-\$ 49,999(n=129,21.6 \%)$. Caregivers had different language preferences (i.e., only English $n=93,15.6 \%$; mostly English $n=184,30.8 \%$; equally English and Spanish $n=271$, $45.3 \%$; mostly Spanish $n=47,7.9 \%$; only Spanish $n=3,0.5 \%$ ) and varied in generational status within the United States (i.e., first $n=145,24.2 \%$; second $n=283,47.3 \%$; third $n=96,16.1 \%$; fourth or higher $n=74,12.4 \%$ ). Caregivers who reported being first-generation in the United States $(n=145)$ were most frequently born in Mexico $(n=37,6.2 \%)$, Venezuela $(n=17,2.8 \%)$, Dominican Republic $(n=18,3 \%)$, Peru $(n=11,1.8 \%)$, Cuba $(n=8,1.3 \%)$, and El Salvador $(n=7,1.2 \%)$. Some first-generation caregivers reported being born in the United States territory of Puerto Rico $(n=43,7.2 \%)$. Youths were 11.9 years old on average $(S D=3.4)$, most commonly boys $(n=328,54.8 \%)$, and largely had health insurance $(n=576,96.3 \%)$. Caregivers reported higher rates of clinically elevated youth internalizing $(n=183,30.6 \%)$ relative to externalizing problems ( $n=134,22.4 \%$; Child Behavior Checklist described in the following section).

\section{Measures}

\section{Demographic Characteristics}

Caregivers were asked to report their demographics (i.e., age, sex, academic attainment, generational status, preferred language, immigration status, household income) and that of their youth (i.e., age, sex, insurance status). 


\section{Barriers to Treatment Questionnaire - Latina/o/x Caregiver}

Barriers to Treatment Questionnaire - Latina/o/x Caregivers (BTQ-LC) is a 24-item measure adapted from the Barriers to Treatment Questionnaire (BTQ) developed by Marques and colleagues (2010). The original BTQ included 23-items asking participants to report how much each factors influenced their decision to delay or avoid seeking MHS in the last year (i.e., logistic/financial; stigma, shame, and discrimination; treatment perception and satisfaction barriers). The original BTQ instructions were adapted to assess factors that influenced caregivers' decisions to delay or avoid seeking support services for their child in the last year. The BTQ-LC retained all 23-items from the original BTQ. Questions were adapted to move the focus from the respondent ("my") to the youth ("my child"). The adaptation also involved the addition of an item assessing fears of deportation that are known to impact MHS utilization among Latina/o/xs (Bridges et al. 2012). Caregivers were provided the following instructions to complete the BTQ-LC: "Please indicate how much each of the following factors influenced your decision to delay or avoid seeking support services for your child in the last year." Responses were: (1) not at all, (2) a little, (3) moderately, (4) very much, (5) extremely. As the original BTQ's factor structure had not been formally examined, two coders independently organized BTQ-LC items into three theorized factors in accordance with constructs and definitions provided by Owens and colleagues (2002): structural, perceptions of mental health problems, perceptions of MHS. Coders had an initial interrater agreement of $91.6 \%$ for item loadings. Disagreements between coders were resolved by discussing disputed items while reviewing the definitions of constructs provided by Owen and colleagues (2002) until agreement was reached. Mean scores were calculated for the global and three factor BTQ-LC scales with higher scores representing greater barriers to seeking youth support services.

\section{Service Need and Utilization}

The Caregiver Support Services Questionnaire (CSSQ) was used to gather information regarding youth MHS that caregivers utilized in the last year (Vázquez and Domenech Rodríguez, 2021). The CSSQ was informed by existing measures of youth service utilization and includes questions assessing frequently used MHS among children (e.g., psychological counseling or therapy, medical doctors, school professionals). The CSSQ was used as established service utilization measures required interview-based administration (Ascher et al. 1996; Bean et al. 2003; Jensen et al. 2004).

\section{Emotional and Behavioral Problems}

The Child Behavior Checklist is 113-item questionnaire that assess a broad array of behavioral problems for youths between ages 6-18 (CBCL; Achenbach and Rescorla 2001). Caregivers reported on the frequency of their child's problem behaviors in the last 6 months: (0) not true, (1) sometimes true, or (2) often true. Two composite scales from CBCL items assess youth internalizing (e.g., depression, anxiety) and externalizing problems (e.g., aggression). Scores on these composite scales can be used to distinguish between youth with clinically elevated internalizing and externalizing (i.e., T-score above 63) and those without. Internal consistency for internalizing $(\alpha=0.95)$ and externalizing $(\alpha=0.96)$ problems scales were 
excellent within the current sample. A dichotomous variable was created to represent youth who had clinically elevated problems on the CBCL (externalizing and/or internalizing) and those that did not.

\section{Analytic Plan}

Statistical analyses were performed using R version 4.0.5 (R Core Team 2021) in the RStudio statistical environment (RStudio Team 2021), with the lavaan (Rosseel 2012), tidyverse (Wickham 2019), psych (Revelle 2019), and furniture (Barrett and Brignone 2017) packages. Common barriers to services impacting youths were examined descriptively using dichotomous indicators for each barrier. Kruskal-Wallis rank sum test were used to determine whether BTQ-LC scores significantly differed between youths with clinical and subclinical problems on the CBCL. Confirmatory factor analysis (CFA) was utilized as it provides an a priori method of testing theoretically derived latent constructs representing barriers to youth MHS (Mueller and Hancock 2001). We used the Diagonally Weighted Least Square estimator as this approach is recommended when examining the factor structure of ordinal data ( $\mathrm{Li}$ 2016). We first constructed a baseline model with all BTQ-LC items loading onto a single global factor. We then fitted the theoretically derived three factor model to compare it to the global factor model. The optimal model was identified based on relative fit indices and their recommended cut offs (Hu and Bentler 1999): Root Mean Square Error of Approximation (RMSEA; good $<0.06$, acceptable $<0.08$, poor fit $>0.10$ ), Comparative Fit Index (CFI; $\geq 0.95$ good fit), Tucker-Lewis Index (TLI; good fit $\geq 0.95$ ), and Standardized Root Mean Square Residual (SRMR; acceptable fit $\leq 0.08$ ). Model selection often requires the researcher's judgement to determine whether a model is meaningfully better than a competing model (Browne and Cudeck 1992). Approximate fit indices are derived from simulations with continuous normally distributed data and are intended to provide a general sense of model fit (Browne and Cudeck 1992; Lai and Green 2016). Models using nonnormative data may be evaluated according to their approximation to these recommended cut offs (Beaujean 2014). Since our data were categorical rather than continuous, we considered the recommended cut off indices flexibly and in the context of additional analyses. For example, we used a scaled chi-square difference test to determine whether the added complexity of the theorized three factor model provided significantly better fit than a single global factor (Brown 2015). Bivariate correlations between BTQ-LC scales and service utilization outcomes were examined in subsequent models.

\section{Results}

\section{Barriers Descriptives}

Within the current sample, Latina/o/x caregivers most frequently endorsed barriers to youth MHS were wanting to address their child's problems on their own ( $n=407,68.1 \%)$, not knowing who to see or where to go for treatment $(n=383,64 \%)$, and feeling that their child's problems were normal for someone in their situation $(n=380,63.5 \%)$. While fears of deportation was the least endorsed item $(n=140,23.4 \%)$, more people endorsed this 
barrier than those who reported being undocumented within the current sample (26 undocumented caregivers $=4.3 \%$ of sample). The most frequent barriers among caregivers of youth with clinically elevated externalizing and/or internalizing problems on the CBCL $(n=211$, $35.3 \%)$ were not knowing who to see or where to go for treatment $(n=278,84.4 \%)$, feeling their child's problems were normal $(n=175,82.9 \%)$, and fear that treatment would be too upsetting for the child $(n=167,79.1 \%)$. The most frequently endorsed items among caregivers of youth with non-clinical problems $(n=387,64.7 \%)$ were wanting to handle their child's problems on their own $(n=242,62.5 \%)$, not knowing who to see or where to go for treatment $(n=205,53 \%)$, and feeling that their child's problems were normal $(n=205$, $53 \%$ ). See Table 1 for dichotomous indicators of barriers by clinical status.

Questionnaire items and scales were found to be positively skewed, which was expected as the sample was non-clinical, and $35.3 \%(n=211)$ of youths reported being within the clinical range for externalizing and/or internalizing problems on the CBCL. The average score for the BTQ-LC global scale was $1.92(S D=0.81)$. Among the subscales, scores on the perceptions regarding mental health problems subscale were the highest $(M=2.02$;

Table 1 Barriers to Youth MHS Among Latina/o/x by Clinical Status

\begin{tabular}{|c|c|c|c|}
\hline & & $\begin{array}{l}\text { Clinical exter } \\
\text { internalizing }\end{array}$ & $\begin{array}{l}\text { lizing and/or } \\
\text { blems }{ }^{1}\end{array}$ \\
\hline & Total & Yes & No \\
\hline & $N=598$ & $\begin{array}{l}(n=211 \\
35.3 \%)\end{array}$ & $\begin{array}{l}(n=387 \\
64.7 \%)\end{array}$ \\
\hline 1) Treatment cost & $351(58.7 \%)$ & $161(76.3 \%)$ & $190(49.1 \%)$ \\
\hline 2) Poor insurance coverage & $333(55.7 \%)$ & $154(73 \%)$ & $179(46.3 \%)$ \\
\hline 3) Did not know how to access services & $383(64 \%)$ & $178(84.4 \%)$ & $205(53 \%)$ \\
\hline 4) Problems with transportation & $214(35.8 \%)$ & $125(59.2 \%)$ & $89(23 \%)$ \\
\hline 5) No time & $287(48 \%)$ & $151(71.6 \%)$ & $136(35.1 \%)$ \\
\hline 6) Lack of appointments & $248(41.5 \%)$ & $139(65.9 \%)$ & $109(28.2 \%)$ \\
\hline 7) No choice of therapist & $317(53 \%)$ & $159(75.4 \%)$ & $158(40.8 \%)$ \\
\hline 8) Wanted to handle problems on their own & $407(68.1 \%)$ & $165(78.2 \%)$ & $242(62.5 \%)$ \\
\hline 9) Embarrassed about child’s problem & $217(36.3 \%)$ & $125(59.2 \%)$ & $92(23.8 \%)$ \\
\hline 10) Embarrassed about needing help & $233(39 \%)$ & $134(63.5 \%)$ & $99(25.6 \%)$ \\
\hline 11) Fear of judgement from friends & $224(37.5 \%)$ & $128(60.7 \%)$ & $96(24.8 \%)$ \\
\hline 12) Fear of judgement from family & $237(39.6 \%)$ & $137(64.9 \%)$ & $100(25.8 \%)$ \\
\hline 13) Fear child would be committed & $246(41.1 \%)$ & $140(66.4 \%)$ & $106(27.4 \%)$ \\
\hline 14) Did not think treatment would help & $260(43.5 \%)$ & $141(66.8 \%)$ & $119(30.7 \%)$ \\
\hline 15) Prior experiences with treatment & $203(33.9 \%)$ & $136(64.5 \%)$ & $67(17.3 \%)$ \\
\hline 16) Unsatisfied with available treatments & $264(44.1 \%)$ & $157(74.4 \%)$ & $107(27.6 \%)$ \\
\hline 17) Afraid treatment would upset child & $299(50 \%)$ & $167(79.1 \%)$ & $132(34.1 \%)$ \\
\hline 18) Do not trust mental health professionals & $250(41.8 \%)$ & $133(63 \%)$ & $117(30.2 \%)$ \\
\hline 19) Difficulty getting motivation & $245(41 \%)$ & $142(67.3 \%)$ & $103(26.6 \%)$ \\
\hline 20) Felt child problems were normal & $380(63.5 \%)$ & $175(82.9 \%)$ & $205(53 \%)$ \\
\hline 21) Fear that child would be discriminated & $242(40.5 \%)$ & $142(67.3 \%)$ & $100(25.8 \%)$ \\
\hline 22) Could not find professional of same race/ethnicity & $201(33.6 \%)$ & $124(58.8 \%)$ & $77(19.9 \%)$ \\
\hline 23) Fear of language barrier & $178(29.8 \%)$ & $102(48.3 \%)$ & $76(19.6 \%)$ \\
\hline 24) Fear of deportation & $140(23.4 \%)$ & $84(39.8 \%)$ & $56(14.5 \%)$ \\
\hline
\end{tabular}

Note: ${ }^{1}$ Clinical problems are defined as scores above the clinical threshold (i.e., T-score above 63 ) on the CBCL internalizing and/or externalizing problems composite scales. 
$S D=0.90)$, followed by structural barriers $(M=1.96 ; S D=0.86)$ and perceptions regarding MHS $(M=1.79 ; S D=0.89)$. Averages on BTQ-LC items and scales are presented in Table 2 for the overall sample. The average score on the global scale among caregivers of youth who were subclinical on the CBCL $(n=387)$ was $1.64(S D=0.68)$. Caregiver of these youth most frequently reported experiencing barriers regarding negative perceptions regarding men-

Table 2 Summary Statistics for BTQ-LC Scales and Items $(\mathrm{N}=598)$

\begin{tabular}{|c|c|c|c|c|}
\hline Items & $\mathrm{M}, \pm \mathrm{SD}$ & Skew & Kurtosis & Alpha \\
\hline Structural & $1.96 \pm 0.86$ & 0.80 & 0.08 & 0.90 \\
\hline 1) I was worried about the cost of treatment. & $2.35 \pm 1.37$ & 0.53 & -1.03 & \\
\hline 2) My health insurance does not cover treatment for my child. & $2.24 \pm 1.33$ & 0.63 & -0.91 & \\
\hline 3) I did not know who to see or where to go for treatment. & $2.42 \pm 1.32$ & 0.41 & -1.07 & \\
\hline $\begin{array}{l}\text { 4) I could not get to treatment because of problems with } \\
\text { transportation. }\end{array}$ & $1.75 \pm 1.15$ & 1.33 & 0.55 & \\
\hline 5) There is no time in my schedule for treatment. & $1.90 \pm 1.12$ & 0.96 & -0.14 & \\
\hline 6) I could not get an appointment. & $1.84 \pm 1.17$ & 1.17 & 0.22 & \\
\hline $\begin{array}{l}\text { 7) I could not choose the person I wanted my child to see for } \\
\text { treatment. }\end{array}$ & $2.05 \pm 1.19$ & 0.80 & -0.48 & \\
\hline 16) I was not satisfied with the services that were available. & $1.85 \pm 1.17$ & 1.23 & 0.40 & \\
\hline $\begin{array}{l}\text { 22) I could not find a mental health professional of my child's } \\
\text { same race or ethnicity. }\end{array}$ & $1.67 \pm 1.08$ & 1.49 & 1.14 & \\
\hline $\begin{array}{l}\text { 23) I was afraid I would not be able to communicate during my } \\
\text { child's treatment because of language barriers. }\end{array}$ & $1.57 \pm 1.01$ & 1.71 & 1.97 & \\
\hline Perceptions of mental health problems & $2.02 \pm 0.90$ & 0.85 & 0.17 & 0.84 \\
\hline 8) I wanted to handle my child's problems on my own. & $2.60 \pm 1.38$ & 0.28 & -1.19 & \\
\hline 9) I felt embarrassed about my child's problems. & $1.75 \pm 1.15$ & 1.35 & 0.66 & \\
\hline $\begin{array}{l}\text { 10) I felt embarrassed about needing help for my child's } \\
\text { problems. }\end{array}$ & $1.82 \pm 1.20$ & 1.24 & 0.31 & \\
\hline $\begin{array}{l}\text { 14) I did not think treatment could help with my child's } \\
\text { problems. }\end{array}$ & $1.82 \pm 1.11$ & 1.17 & 0.28 & \\
\hline $\begin{array}{l}\text { 19) I had difficulty motivating myself to seek treatment for my } \\
\text { child. }\end{array}$ & $1.79 \pm 1.11$ & 1.21 & 0.37 & \\
\hline $\begin{array}{l}\text { 20) I felt like my child's problems were normal for someone in } \\
\text { their situation. }\end{array}$ & $2.31 \pm 1.29$ & 0.60 & -0.82 & \\
\hline Perceptions of mental health services & $1.79 \pm 0.89$ & 1.08 & 0.39 & 0.90 \\
\hline $\begin{array}{l}\text { 11) I was worried about being judged or criticized by my friends } \\
\text { if I sought treatment for my child. }\end{array}$ & $1.80 \pm 1.19$ & 1.24 & 0.30 & \\
\hline $\begin{array}{l}\text { 12) I was worried about being judged or criticized by my family } \\
\text { if I sought treatment for my child. }\end{array}$ & $1.82 \pm 1.18$ & 1.19 & 0.23 & \\
\hline $\begin{array}{l}\text { 13) I was afraid that my child would be committed to a hospital } \\
\text { against my will. }\end{array}$ & $1.91 \pm 1.29$ & 1.19 & 0.10 & \\
\hline $\begin{array}{l}\text { 15) My child received treatment before, and it did not help with } \\
\text { their problems. }\end{array}$ & $1.70 \pm 1.11$ & 1.41 & 0.83 & \\
\hline 17) I was afraid treatment for my child would be too upsetting. & $2.01 \pm 1.22$ & 0.96 & -0.20 & \\
\hline 18) I don't trust mental health professionals. & $1.78 \pm 1.09$ & 1.25 & 0.59 & \\
\hline $\begin{array}{l}\text { 21) I was afraid my child would be treated badly in treatment } \\
\text { because of their race or ethnicity. }\end{array}$ & $1.84 \pm 1.19$ & 1.18 & 0.21 & \\
\hline $\begin{array}{l}\text { 24) I was afraid of being deported for seeking treatment for my } \\
\text { child. }\end{array}$ & $1.49 \pm 0.99$ & 1.89 & 2.42 & \\
\hline Global & $1.92 \pm 0.81$ & 0.96 & 0.51 & 0.95 \\
\hline
\end{tabular}


tal health problems $(M=1.76 ; S D=0.78)$. These families also reported experiencing more structural barriers $(M=1.69 ; S D=0.76)$ relative to negative perceptions of MHS $(M=1.48$; $S D=0.70$ ). Among the 211 youth who had clinically elevated problems on the CBCL, the mean score for the global scale was significantly higher than the average among subclinical youths $(M=2.44 ; S D=0.77 ; H[1]=151.87, p<.001)$. All subscale scores were significantly higher among caregivers of youth who were within the clinical range on the CBCL: structural barriers $(M=2.47 ; S D=0.80 ; H[1]=127.22, p<.001)$, perceptions regarding mental health problems $(M=2.48 ; S D=0.94 ; H[1]=87.81, p<.001)$, and perceptions regarding MHS $(M=2.37 ; S D=0.91 ; H[1]=150.57, p<.001)$.

\section{Psychometrics}

The internal consistency for each BTQ-LC scale was assessed using Cronbach's alpha. Internal consistency for the global scale was excellent $(\alpha=0.95)$. Internal consistency for BTQLC proposed subscales ranged from good to excellent (i.e., structural barriers $[\alpha=0.90]$, perceptions of mental health problems $[\alpha=0.84]$ and services $[\alpha=0.90])$.

Table 3 Fit Statistics for Baseline and 3 Factor Models $(\mathrm{N}=598)$

\begin{tabular}{lllllll}
\hline & $X^{2}$ & $d f$ & $\begin{array}{l}\text { RMSEA } \\
{[90 \% \text { CI }]}\end{array}$ & CFI & TLI & SRMR \\
\hline 1 Factor 24 Items & 2254.23 & 252 & $0.115[0.111-0.120]^{*}$ & 0.983 & 0.981 & 0.083 \\
3 Factor 24 Items $^{*}$ Factor+Outcomes $^{\text {a }}$ & 33755.50 & 249 & $0.101[0.096-0.105] *$ & 0.987 & 0.986 & 0.073 \\
3 Factor+Outcomes $^{\text {a }}$ & 2837.34 & 315 & $0.116[0.112-0.120]^{*}$ & 0.979 & 0.977 & 0.096 \\
\hline
\end{tabular}

Note: $p<.001 *$ a Bivariate correlations between BTQ-LC scale(s) and service utilization outcomes

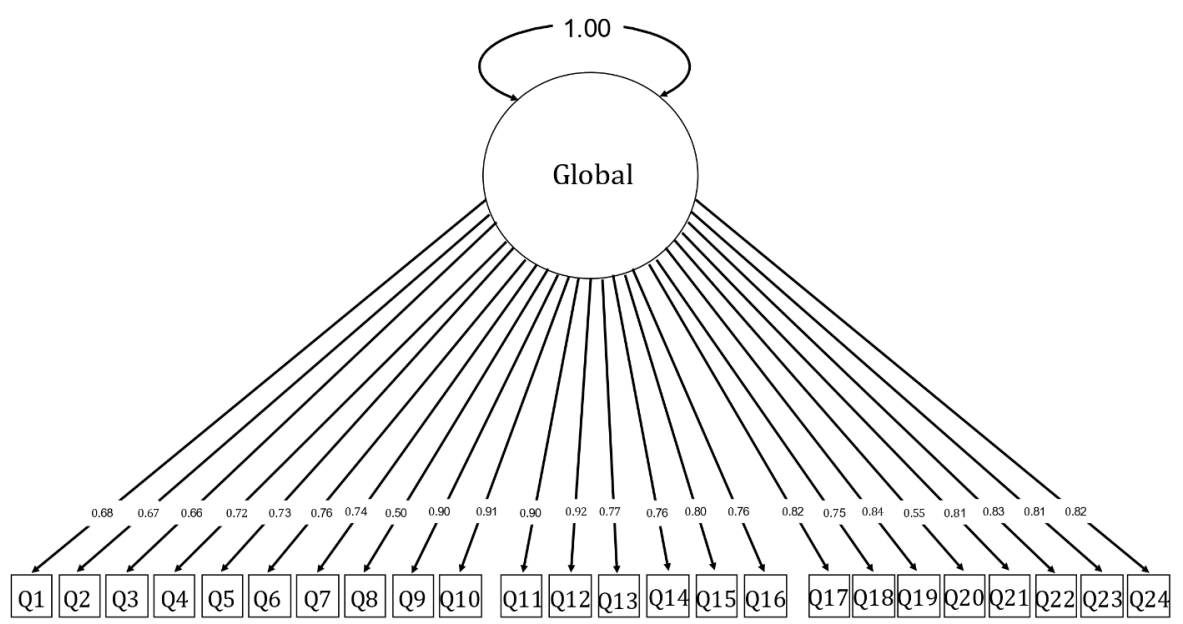

Fig. 1 Barriers to Treatment Questionnaire - Latina/o/x Caregivers Global Model $(\mathrm{N}=598)$ 
The global model CFA had mixed results across relative fit indices. Metrics such as RMSEA (0.115) and SRMR (0.083) suggested a poor model fit. In contrast, CFI (0.983) and TLI (0.981) indices fit the data well. See Table 3 for all model fit indices for the global and three-factor models. It should be noted that the SRMR approached the recommended cut off for acceptable model fit for the global solution $(\leq 0.08$; Hu and Bentler 1999). The theorized three factor model demonstrated small improvements in CFI (0.987) and TLI (0.986) metrics. The SRMR (0.073) index was within the acceptable range for the three-factor model. While RMSEA improved relative to the global model, this index suggested that the threefactor model fit the data poorly (0.101). A scaled chi-square difference test found that the added complexity of the theorized three-factor model significantly improved fit relative to a single global factor $X^{2}$ (ChisqDif[3] $=257.57, p<.001$ ). This finding suggest that the BTQLC may be best represented by three subscales rather than a single global scale. All items loaded onto their respective global scale and subscales and met the factor loading threshold of 0.40 . See Figs. 1 and 2 for factor loadings for the global and subscale models.

\section{Utilization Outcomes}

Bivariate correlations were conducted between service utilization outcomes (i.e., psychological counseling $[n=210 ; 35.1 \%]$, school professional $[n=222 ; 37.1 \%]$, and medical doctor $[n=258 ; 43.1 \%])$ and BTQ-LC scales. The BTQ-LC global scale was negatively associated with utilization of psychological counseling $(r=-.39, p<.001)$, school professionals $(r=-.26, p<.001)$, and medical doctors $(r=-.25, p<.001)$. The structural barriers subscale was negatively associated with utilization of psychological counseling $(r=-.43, p<.001)$, school professionals $(r=-.27, p<.001)$, and medical doctors $(r=-.29, p<.001)$, such that those who experienced greater structural barriers were less likely to seek out services. The perceptions regarding mental health problems subscale was negatively related to utilization of psychological counseling $(r=-.26, p<.001)$, school professionals $(r=-.18, p=.001)$,

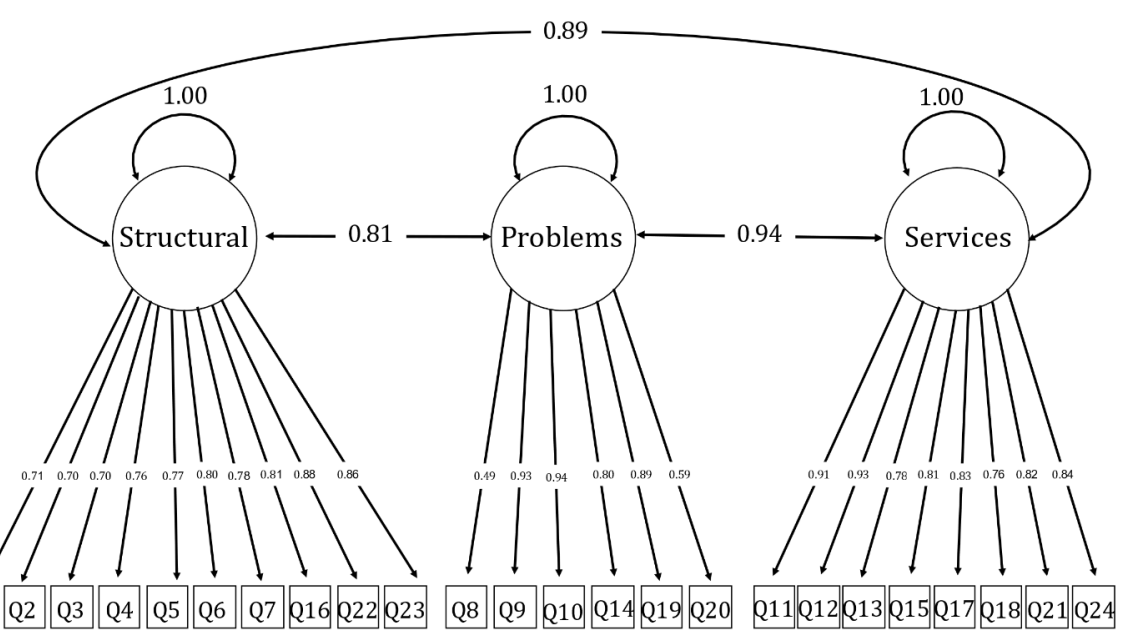

Fig. 2 Barriers to Treatment Questionnaire - Latina/o/x Caregivers Three Factor Model $(\mathrm{N}=598)$ 
and medical doctors $(r=-.16, p=.005)$, such that higher scores were associated with lower odds of service utilization. Lastly, the perceptions of MHS subscale was also negatively correlated with utilization of psychological counseling $(r=-.39, p<.001)$, school professionals $(r=-.27, p<.001)$, and medical doctors $(r=-.26, p<.001)$, such that more negative perceptions were associated with lower odds of service utilization. See Table 3 for model fit indices for global and three-factor models with bivariate correlations.

\section{Discussion}

The current study validated a brief barriers-to-treatment questionnaire to facilitate quantitative analysis of factors contributing to the underutilization of youth MHS among Latina/o/ xs. Our findings suggest that the three-factor model based on a priori theoretical guidance by Owens and colleagues (2002) demonstrated better model fit relative to a single global scale across multiple fit indices (i.e., RMSEA, CFI, TLI, SRMR). However, RMSEA for both models were within the poor range and SRMR was only within the acceptable range for the three-factor model. Inconsistencies between approximate fit indices are common and interpretations often require a degree of judgement on the part of the researcher to determine whether a model is meaningfully better than a competing model (Browne and Cudeck 1992; Lai and Green 2016). While a scaled chi-square test found that the theorized three-factor model had a superior fit relative to a single factor, the CFI and TFI for these subscales were only marginally higher than those of the global factor. The SRMR index also approached acceptability for the global scale (0.083; acceptable $\leq 0.080$; Hu and Bentler 1999). These findings and strong internal consistency across scales may suggest that the BTQ-LC could be represented by both the theorized three-factor model and a single global scale. Construct validity was established as caregiver scores on BTQ-LC scales (i.e., global and three factor) were negatively correlated with the utilization of common youth MHS (i.e., psychological counseling, school professionals, medical doctors; Duong et al. 2020). These findings suggest that the BTQ-LC could be used to support population-level research that has not been possible due to the absence of an existing validated measure.

This measure is not intended to replace interview-based instruments that provide rich information regarding challenges faced by Latina/o/x families. Instead, the BTQ-LC aims to provide an additional tool that can be used to quantify barriers to youth MHS among Latina/o/xs. Qualitative research often utilizes intensive interviews and/or thematic analysis, which is time and resource consuming, limiting the number of participants that can be surveyed (Chapman and Stein 2014; Gerdes et al. 2014). Utilizing a self-report measure like the BTQ-LC could allow researchers to survey a greater number of participants, which may improve the generalizability of findings and facilitate more advanced statistical analyses. This is needed as current research examining factors contributing to Latina/o/x caregivers' utilization of youth MHS lack covariates representing barriers to care, potentially overlooking important influences when building explanatory models to understand mental health disparities (Finno-Velasquez et al. 2016; Galvan and Gudiño 2021). Furthermore, BTQ-LC scales may help elucidate the extent to which specific clusters of barriers (i.e., structural, perceptions of problems, perceptions of services) reduce the odds that Latina/o/x families will engage in youth MHS. Understanding barriers to service utilization is relevant and 
timely given the escalating incidence of youth mental illness (Twenge et al. 2019) and the costs of having youth mental illness go untreated (Trautmann et al. 2016).

Gathering data regarding a wide variety of barriers can also help identify spcific targets for reducing mental health disparities. Descriptive findings from the present study highlight potential avenues to reduce treatment barriers among Latina/o/x families. Our findings support the notion of considering caregiver perception of mental health problems (Kapke and Gerdes 2016), as caregivers on average had higher ratings of perceptions regarding mental health problems relative to other barriers (i.e., perceptions of MHS; structural barriers). Caregivers' negative perceptions of mental health problems are mutable and can be address through psychoeducation (Srebnik et al. 1996). Descriptive analysis of dichotomous indicators representing BTQ-LC items also identified specific common barriers to accessing youth MHS. Prior research has documented that Latina/o/x caregivers are more likely to normalize youth psychopathology and often report difficulties locating and accessing services (Raglin Bignall et al. 2015; Chapman and Stein 2014). Our findings expand this work by highlighting the relative importance of these factors as they were among the most frequently endorsed barriers among caregivers of youth with clinically elevated problems on the CBCL. Interestingly, while fear of deportation was the least frequently endorsed barrier to care, the number of caregivers that endorsed this item represented nearly one quarter of the sample and, notably, exceed the number of individuals that reported being undocumented. This information is consistent with findings that suggest that stress related to documentation spills over into communities (Rhodes et al. 2015). It is also possible that this may reflect a fear of deportation of relatives in mixed-status families (i.e., mixed citizenship and immigration statuses). Mixed-status families have reported reluctancy in seeking health care services due to the impact that it may have on undocumented family members (Castañeda and Melo 2014).

The BTQ-LC displayed greater internal consistency for the global scale relative to prior versions used on Black and White participants (Marques et al. 2010; Williams et al. 2012). However, prior versions of the questionnaire were not developed to assess caregiver barriers to youth MHS utilization. Further adaptation to the BTQ-LC may be needed to facilitate the examination of barriers to youth MHS within or between other underserved groups.

\section{Limitations}

There are some important limitations that must be considered to contextualize our findings. Our data are cross-sectional and cannot be used to establish a causal relationship between barriers and service utilization outcomes. Establishing temporal relationships would be critical to informing interventions and improving the accessibility of youth MHS. The current study utilized data from participants that provided complete responses, which may have biased our results. It is possible that participants may have been less likely to complete the survey if they felt uncomfortable with questions regarding their youth's mental health problems. While participants were offered the option "prefer not to answer" on the deportation question, it is possible that this item may have contributed to participant attrition due to fears of persecution. Examining patterns of missingness and utilizing imputation methods was not possible as Qualtrics only provided data on cases with complete high-quality responses. Future research should replicate our findings using datasets where all respondents provided 
complete data or in situations in which robust imputation methods can be used. The current study gathered data during the coronavirus pandemic. It is possible that stay-at-home orders may have exacerbated or contributed to barriers that caregivers reported within the current study (Vázquez et al., 2021b).

The Latina/o/x population in the United States is diverse in national origins, ethnic and cultural traditions, and languages (Noe-Bustamante 2019). The BTQ-LC was validated with English speaking individuals as a first step. Participants within the current sample were largely bicultural as indicated by their equal preference towards speaking English and Spanish $(n=271 ; 45.3 \%)$ and many caregivers were the first generation in the United States $(n=145 ; 24.2 \%)$. This is an important limitation of the current study as Latina/o/x caregivers who primarily speak Spanish may experience more language-based barriers (e.g., discrimination related to language/accent; D'Anna et al. 2010) and other obstacles related to acculturation that could impact youth MHS utilization (e.g., preferences for informal supports; Garland et al. 2005). Nonetheless, researchers working with Latina/o/x populations might extend this important work by validating a Spanish version of the BTQ-LC. To facilitate this work, we have provided access to a Spanish version of the measure (osf.io/ gpk4s) which has undergone a translation, backtranslation, and bilingual committee review process. Future research should also consider adding additional barriers to the BTQ-LC to reflect challenges related to family support for seeking youth MHS and need for childcare (Kapke and Gerdes 2016). Despite these limitations, the BTQ-LC provides a potentially useful quantitative measure for examining barriers to youth MHS within an understudied population.

\section{Conclusions}

A lack of validated tools may be implicated in the dearth of information regarding barriers that are most commonly experienced by Latina/o/x families. The BTQ-LC represents an important resource so that large-scale quantitative research may be conducted to further understand mental health disparities among Latina/o/x populations. The BTQ-LC represents an important step towards improving access to established questionnaires needed to study mental health disparities among Latina/o/x youths. Future research may explore Latina/o/x families' barriers profiles using the BTQ-LC to learn whether varied clusters of barriers differentially contribute to underutilization of MHS. Overall, the BTQ-LC represents a novel measure of barriers to service utilization for use with Latina/o/x caregivers to examine patterns of youth MHS utilization.

Authors' contributions All authors have contributed equally to the development of this research and this manuscript.

Funding This research was funded by an American Psychological Foundation Visionary Fund Grant (\# 202803). PI: Alejandro L. Vázquez.

Availability of data and material The lead author, Alejandro L. Vázquez, takes responsibility for the integrity of the data and accuracy of the data analysis. Data are not publicly available but can be requested from the lead author to confirm the findings of the current study. Materials are made available upon request. 
Code availability The current manuscript conducted analysis using $\mathrm{r}$ mark down. Code is provided upon request.

\section{Declarations}

Conflicts of interest/Competing interests The authors have no relevant financial or non-financial interests to disclose.

Ethics approval We have complied with all ethical guidelines established by the American Psychological Association and obtained human subjects' approval from the [blinded for review] Institutional Review Board prior to data collection. All research actives were performed in accordance with the ethical standards articulated in the 1964 Declaration of Helsinki, its later amendments, and the 1979 Belmont Report.

Consent to participate All participant provided informed consent to participate in the current study prior to data collection.

Consent for publication All participant provided informed consent to utilize deidentified data for publication. All authors have agreed with the manuscripts content and have consented to submitting it for publication.

\section{References}

Abbey, J. D., \& Meloy, M. G. (2017). Attention by design: Using attention checks to detect inattentive respondents and improve data quality. Journal of Operations Management, 53, 63-70. https://doi. org/10.1016/j.jom.2017.06.001

Achenbach, T. M., \& Rescorla, L. A. (2001). Manual for the ASEBA School-Age Forms \& Profiles. University of Vermont, Research Center for Children, Youth, \& Families

Ascher, B. H., Farmer, Z., Burns, E. M., \& Angold, A., B. J., \&. (1996). The child and adolescent services assessment (CASA) description and psychometrics. Journal of Emotional and Behavioral Disorders, 4(1), 12-20. https://doi.org/10.1177/106342669600400102

Barrett, T., \& Brignone, E. (2017). Furniture for Quantitative Scientists. The R Journal, 9, 142-148. https:// doi.org/10.32614/rj-2017-037

Bean, D. L., Rotheram-Borus, M. J., Leibowitz, A., Horwitz, S. M., \& Weidmer, B. (2003). Spanish-language services assessment for children and adolescents (SACA): reliability of parent and adolescent reports. Journal of the American Academy of Child \& Adolescent Psychiatry, 42(2), 241-248. https:// doi.org/10.1097/00004583-200302000-00019

Beaujean, A. A. (2014). Latent variable modeling using R: A step-by-step guide. Routledge

Berger-Jenkins, E., McKay, M., Newcorn, J., Bannon, W., \& Laraque, D. (2012). Parent medication concerns predict underutilization of mental health services for minority children with ADHD. Clinical Pediatrics, 51(1), 65-76. https://doi.org/10.1177/0009922811417286

Bridges, A. J., Andrews, I. I. I., \& Deen, T. L., A. R., \&. (2012). Mental health needs and service utilization by Hispanic immigrants residing in mid-southern United States. Journal of Transcultural Nursing, 23(4), 359-368. https://doi.org/10.1177/1043659612451259

Brown, T. A. (2015). Confirmatory factor analysis for applied research (2nd ed.). Guilford publications

Browne, M. W., \& Cudeck, R. (1992). Alternative ways of assessing model fit. Sociological Methods \& Research, 21(2), 230-258. https://doi.org/10.1177/0049124192021002005

Castañeda, H., \& Melo, M. A. (2014). Health care access for Latino mixed-status families: Barriers, strategies, and implications for reform. American Behavioral Scientist, 58(14), 1891-1909. https://doi.org/1 0.1177/0002764214550290https://doi-org.dist.lib.usu.edu/

Cauce, A. M. (2011). Is multicultural psychology a-scientific?: Diverse methods for diversity research. Cultural Diversity and Ethnic Minority Psychology, 17(3), 228-233. https://doi.org/10.1037/a0023880

Cauce, A. M., Domenech-Rodríguez, M., Paradise, M., Cochran, B. N., Shea, J. M., Srebnik, D., \& Baydar, N. (2002). Cultural and contextual influences in mental health help seeking: a focus on ethnic minority youth. Journal of Consulting and Clinical Psychology, 70(1), 44-55. https://doi. org/10.1037/0022-006x.70.1.44

Chapman, M. V., \& Stein, G. L. (2014). How do new immigrant Latino parents interpret problem behavior in adolescents? Qualitative Social Work, 13(2), 270-287. https://doi.org/10.1177/1473325012468478 
D’Anna, L. H., Ponce, N. A., \& Siegel, J. M. (2010). Racial and ethnic health disparities: Evidence of discrimination's effects across the SEP spectrum. Ethnicity \& Health, 15(2),121-143. https://doi. org/10.1080/13557850903490298

De Silva, L. E. D., Ponting, C., Ramos, G., Guevara, M. V. C., \& Chavira, D. A. (2020). Urban Latinx parents' attitudes towards mental health: Mental health literacy and service use. Children and Youth Services Review, 109, 104719. https://doi.org/10.1016/j.childyouth.2019.104719

Duong, M. T., Bruns, E. J., Lee, K., Cox, S., Coifman, J., Mayworm, A., \& Lyon, A. R. (2020). Rates of mental health service utilization by children and adolescents in schools and other common service settings: A systematic review and meta-analysis. Administration and Policy in Mental Health and Mental Health Services Research, 48(3), 420-439. https://doi.org/10.1007/s10488-020-01080-9

Finno-Velasquez, M., Cardoso, J. B., Dettlaff, A. J., \& Hurlburt, M. S. (2016). Effects of parent immigration status on mental health service use among Latino children referred to child welfare. Psychiatric Services, 67(2), 192-198. https://doi.org/10.1176/appi.ps.201400444

Galvan, T., \& Gudiño, O. G. (2021). Understanding Latinx youth mental health disparities by problem type: The role of caregiver culture. Psychological Services, 18(1), 116-123. https://doi.org/10.1037/ ser0000365

Garland, A. F., Lau, A. S., Yeh, M., McCabe, K. M., Hough, R. L., \& Landsverk, J. A. (2005). Racial and ethnic differences in utilization of mental health services among high-risk youths. American Journal of Psychiatry, 162(7), 1336-1343. https://doi.org/10.1176/appi.ajp.162.7.1336

Gerdes, A. C., Lawton, K. E., Haack, L. M., \& Schneider, B. W. (2014). Latino parental help seeking for childhood ADHD. Administration and Policy in Mental Health and Mental Health Services Research, 41(4), 503-513. https://doi.org/10.1007/s10488-013-0487-3

Hu, L. T., \& Bentler, P. M. (1999). Cutoff criteria for fit indexes in covariance structure analysis: Conventional criteria versus new alternatives. Structural Equation Modeling: A Multidisciplinary Journal, 6(1), 1-55. https://doi.org/10.1080/10705519909540118

Jensen, P. S., Hoagwood, E., Roper, K., Arnold, M., Odbert, L. E., Crowe, C., \& Wells, M., K. (2004). The services for children and adolescents-parent interview: Development and performance characteristics. Journal of the American Academy of Child and Adolescent Psychiatry, 43(11), 1334-1344. https://doi. org/10.1097/01.chi.0000139557.16830.4e

Kapke, T. L., \& Gerdes, A. C. (2016). Latino family participation in youth mental health services: Treatment retention, engagement, and response. Clinical Child and Family Psychology Review, 19(4), 329-351. https://doi.org/10.1007/s10567-016-0213-2

Kataoka, S. H., Zhang, L., \& Wells, K. B. (2002). Unmet need for mental health care among U.S. children: Variation by ethnicity and insurance status. The American Journal of Psychiatry, 159(9), 1548-1555. https://doi.org/10.1176/appi.ajp.159.9.1548

Kazdin, A. E., Holland, L., Crowley, M., \& Breton, S. (1997). Barriers to treatment participation scale: Evaluation and validation in the context of child outpatient treatment. Journal of Child Psychology and Psychiatry, 38(8), 1051-1062. https://doi.org/10.1111/j.1469-7610.1997.tb01621.x

Kennedy, R., Clifford, S., Burleigh, T., Waggoner, P. D., Jewell, R., \& Winter, N. J. (2020). The shape of and solutions to the MTurk quality crisis. Political Science Research and Methods, 8(4), 614-629. https:// doi.org/10.1017/psrm.2020.6

Lai, K., \& Green, S. B. (2016). The problem with having two watches: Assessment of fit when RMSEA and CFI disagree. Multivariate Behavioral Research, 51(2-3), 220-239. https://doi.org/10.1080/00273171 .2015 .1134306

Li, C. H. (2016). Confirmatory factor analysis with ordinal data: Comparing robust maximum likelihood and diagonally weighted least squares. Behavior Research Methods, 48(3), 936-949. https://doi. org/10.3758/s13428-015-0619-7

Lowry, P. B., D’Arcy, J., Hammer, B., \& Moody, G. D. (2016). "Cargo Cult” science in traditional organization and information systems survey research: A case for using nontraditional methods of data collection, including Mechanical Turk and online panels. The Journal of Strategic Information Systems, 25(3), 232-240. https://doi.org/10.1016/j.jsis.2016.06.002

Malhotra, K., Shim, R., Baltrus, P., Heiman, H. J., Adekeye, O., \& Rust, G. (2015). Racial/ethnic disparities in mental health service utilization among youth participating in negative externalizing behaviors. Ethnicity \& Disease, 25(2), 123-129. Retrieved from https:/ethndis.org/edonline/index.php/ethndis/ article/view/117

Marques, L., LeBlanc, N. J., Weingarden, H. M., Timpano, K. R., Jenike, M., \& Wilhelm, S. (2010). Barriers to treatment and service utilization in an internet sample of individuals with obsessive-compulsive symptoms. Depression and Anxiety, 27(5), 470-475. https://doi.org/10.1002/da.20694 
Merikangas, K. R., He, J., Burstein, M., Swendsen, J., Avenevoli, S., Case, B., \& Olfson, M. (2011). Service utilization for lifetime mental disorders in U.S. adolescents: Results of the National Comorbidity Survey-Adolescent Supplement (NCS-A). Journal of the American Academy of Child \& Adolescent Psychiatry, 50(1), 32-45. https://doi.org/10.1016/j.jaac.2010.10.006

Mueller, R. O., \& Hancock, G. R. (2001). Factor analysis and latent structure, confirmatory factor alaysis. In N. J. Smelser, \& P. B. Baltes (Eds.), International Encyclopedia of the Social \& Behavioral Sciences (pp. 5239e5244). Oxford, UK: Elsevier. https://doi.org/10.1016/b0-08-043076-7/00426-5

Noe-Bustamante, L. (2019, September 16). Key facts about U.S. Hispanics and their diverse heritage. Retrieved from https://www.pewresearch.org/fact-tank/2019/09/16/key-facts-about-u-s-hispanics/

Owens, P. L., Hoagwood, K., Horwitz, S. M., Leaf, P. J., Poduska, J. M., Kellam, S. G., \& Ialongo, N. S. (2002). Barriers to children's mental health services. Journal of the American Academy of Child \& Adolescent Psychiatry, 41(6), 731-738. https://doi.org/10.1097/00004583-200206000-00013

Pepin, R., Segal, D. L., \& Coolidge, F. L. (2009). Intrinsic and extrinsic barriers to mental health care among community-dwelling younger and older adults. Aging \& Mental Health, 13(5), 769-777. https://doi. org/10.1080/13607860902918231

R Core Team. (2021). R: A language and environment for statistical computing. R Foundation for Statistical Computing, https://www.R-project.org/

Raglin Bignall, W. J., Jacquez, F., \& Vaughn, L. M. (2015). Attributions of mental illness: An ethnically diverse community perspective. Community Mental Health Journal, 51(5),540-545. https://doi. org/10.1007/s10597-014-9820-x

Reardon, T., Harvey, K., Baranowska, M., O’Brien, D., Smith, L., \& Creswell, C. (2017). What do parents perceive are the barriers and facilitators to accessing psychological treatment for mental health problems in children and adolescents? A systematic review of qualitative and quantitative studies. European Child \& Adolescent Psychiatry, 26(6), 623-647. https://doi.org/10.1007/s00787-016-0930-6

Revelle, W. (2019). psych: Procedures for personality and psychological research.https://CRAN.R-project. org/package $=$ psych

Rhodes, S. D., Mann, L., Simán, F. M., Song, E., Alonzo, J., Downs, M., \& Hall, M. A. (2015). The impact of local immigration enforcement policies on the health of immigrant Hispanics/Latinos in the United States. American Journal of Public Health, 105(2), 329-337. https://doi.org/10.2105/ajph.2014.302218

Rosseel, Y. (2012). lavaan: An R Package for Structural Equation Modeling. Journal of Statistical Software, 48, 1-36. https://doi.org/10.18637/jss.v048.i02

RStudio, T. (2021). RStudio: Integrated Development for $R$. http://www.rstudio.com/

Scheppers, E., Van Dongen, E., Dekker, J., Geertzen, J., \& Dekker, J. (2006). Potential barriers to the use of health services among ethnic minorities: A review. Family Practice, 23(3), 325-348. https://doi. org/10.1093/fampra/cmi113

Seid, M., Opipari-Arrigan, L., Gelhard, L. R., Varni, J. W., \& Driscoll, K. (2009). Barriers to care questionnaire: Reliability, validity, and responsiveness to change among parents of children with asthma. Academic Pediatrics, 9(2), 106-113. https://doi.org/10.1016/j.acap.2008.12.003

Srebnik, D., Cauce, A. M., \& Baydar, N. (1996). Help-seeking pathways for children and adolescents. Journal of Emotional and Behavioral Disorders, 4(4), 210-220. https://doi.org/10.1177/106342669600400402

Trautmann, S., Rehm, J., \& Wittchen, H. U. (2016). The economic costs of mental disorders: Do our societies react appropriately to the burden of mental disorders? EMBO reports, 17(9), 1245-1249. https://doi. org/10.15252/embr.201642951

Tsang, Y. T., Franklin, M., Sala-Hamrick, K., Kohlberger, B., Simon, V. A., Partridge, T., \& Barnett, D. (2020). Caregivers as gatekeepers: Professional mental health service use among urban minority adolescents. American Journal of Orthopsychiatry, 90(3), 328-339. https://doi.org/10.1037/ort0000432

Twenge, J. M., Cooper, A. B., Joiner, T. E., Duffy, M. E., \& Binau, S. G. (2019). Age, period, and cohort trends in mood disorder indicators and suicide-related outcomes in a nationally representative dataset, 2005-2017. Journal of Abnormal Psychology, 128(3), 185-199. https://doi.org/10.1037/abn0000410

Vázquez, A. L., \& Domenech Rodríguez, M. M. (2021). Caregiver support services questionnaire (CSSQ). Retrieved from https://osf.io/ya4ge

Vázquez, A. L., Alvarez, M. C., Navarro Flores, C. M., González Vera, J. M., Barrett, T. S., \& Domenech Rodríguez, M. M. (2021a). Youth mental health service preferences and utilization patterns among Latinx caregivers. Children and Youth Services Review. Advanced online publication. https://doi. org/10.1016/j.childyouth.2021.106258

Vázquez, A. L., Navarro Flores, C. M., Alvarez, M. C., \& Domenech Rodríguez, M. M. (2021b). Latinx caregivers? perceived need for and utilization of youth telepsychology services during the Coronavirus pandemic. Journal of Latinx Psychology. Advanced online publication. https://doi.org/10.1037/lat0000192

Villagrana, M. (2010). Mental health services for children and youth in the child welfare system: A focus on caregivers as gatekeepers. Children and Youth Services Review, 32(5), 691-697. https://doi. org/10.1016/j.childyouth.2010.01.005 
Wickham, H. (2019). Welcome to the Tidyverse. Journal of Open Source Software, 4, 1-6. https://doi. org/10.21105/joss.01686

Williams, M. T., Domanico, J., Marques, L., Leblanc, N. J., \& Turkheimer, E. (2012). Barriers to treatment among African Americans with obsessive-compulsive disorder. Journal of Anxiety Disorders, 26(4), 555-563. https://doi.org/10.1016/j.janxdis.2012.02.009

Publisher's Note Springer Nature remains neutral with regard to jurisdictional claims in published maps and institutional affiliations. 